PROCEEDINGS OF THE

AMERICAN MATHEMATICAL SOCIETY

Volume 127, Number 2, February 1999, Pages 323-325

S 0002-9939(99)04515-3

\title{
METRIZABLE AND $\mathbb{R}$-METRIZABLE BETWEENNESS SPACES
}

\author{
JURAJ ŠIMKO \\ (Communicated by Carl G. Jockusch, Jr.)
}

\begin{abstract}
It is proved that the theory of the class of all betweenness spaces metrizable by real-valued metrics does not coincide with the theory of the class of all betweenness spaces metrizable by metrics taking values in any ordered field. This solves a problem raised by Mendris and Zlatoš.
\end{abstract}

Let $d$ be a metric on a nonempty set $A$ taking values in an ordered field $F$. The ternary betweenness relation $T_{d}$ on $A$ is defined by

$$
T_{d}(x, y, z) \Leftrightarrow d(x, y)+d(y, z)=d(x, z) .
$$

A first-order structure $(A, T)$ with a single ternary relation $T$ is called a metrizable betweenness space if $T=T_{d}$ for some metric $d$ on $A$; it is called an $\mathbb{R}$-metrizable betweenness space if $T=T_{d}$ for some real-valued $d$.

As proved by $\mathrm{R}$. Mendris and P. Zlatoš in [1], the class $\mathcal{M}$ of all metrizable betweenness spaces, i.e., the class of all first-order structures of the form $\left(A, T_{d}\right)$, where $d$ is a metric on $A$, is a universal elementary one. On the other hand, being not closed under elementary extensions, the class $\mathcal{M}_{0}$ of all $\mathbb{R}$-metrizable betweenness spaces is not elementary. However, the question of whether $\mathcal{M}$ is the least elementary class containing $\mathcal{M}_{0}$ or, equivalently, the question of whether $\operatorname{Th} \mathcal{M}_{0}=\operatorname{Th} \mathcal{M}$ remained open. In this short note we will answer that question negatively.

Let $|x y| \leqslant|u v|$ stand as the abbreviation for the formula

$$
(T(x, y, v) \& T(u, x, y)) \vee(T(x, y, u) \& T(v, x, y)) .
$$

Obviously, in a metrizable betweenness space $\left(A, T_{d}\right),|x y| \leqslant|u v|$ implies $d(x, y) \leqslant$ $d(u, v)$ for any $x, y, u, v \in A$.

Further, let $\phi(x, y, z, u, v)$ be the formula expressing that $x, y, z, u, v$ are five distinct elements, and $T(x, y, z), T(u, x, y), T(u, z, v)$ are the only non-trivial betweenness relations among them.

Finally, let us denote by $\theta$ the sentence:

$$
\begin{aligned}
&(\exists x, y, z, u, v) \phi(x, y, z, u, v) \&(\forall x, y, z, u, v)[\phi(x, y, z, u, v) \\
&\left.\Rightarrow\left(\left(\exists z^{\prime}\right)\left(z^{\prime} \neq v \& T\left(z, z^{\prime}, v\right) \&|x y| \leqslant\left|z z^{\prime}\right|\right)\right)\right] .
\end{aligned}
$$

Theorem 1. $\neg \theta \in \operatorname{Th} \mathcal{M}_{0} \backslash \operatorname{Th} \mathcal{M}$; consequently, the least elementary class containing $\mathcal{M}_{0}$ is a proper subclass of $\mathcal{M}$.

Received by the editors April 2, 1997 and, in revised form, May 15, 1997.

1991 Mathematics Subject Classification. Primary 03B30, 03C52, 51F99.

Key words and phrases. Betweenness relation, metric, ordered field, elementary class.

(C)1999 American Mathematical Society 


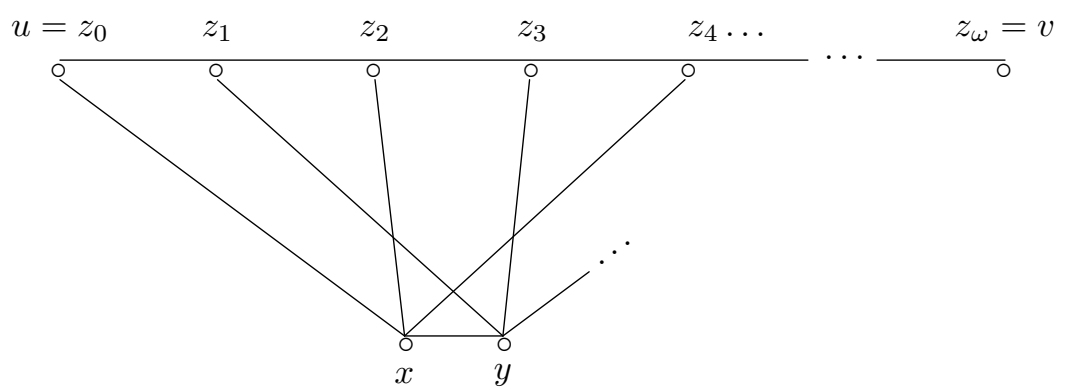

Diagram 1

Proof. (1) $\neg \theta \in \operatorname{Th} \mathcal{M}_{0}$ : Let $(A, T)$ be a metrizable betweenness space satisfying $\theta$, let $d$ be any metric on $A$ inducing $T$, and let $x, y, z, u, v$ be the elements of $A$ guaranteed by $\theta$. By induction one can construct a sequence $\left(z_{n}\right)_{n<\omega}$ in $A$, such that $z_{0}=u, z_{1}=z$ and $z_{n+1}=z_{n}^{\prime}$ for $n \geqslant 1$, i.e., $z_{n+1} \neq v, T\left(z_{n}, z_{n+1}, v\right)$ and $|x y| \leqslant\left|z_{n} z_{n+1}\right|$. Then both $T\left(u, z_{m}, z_{n}\right)$ and $T\left(z_{m}, z_{n}, v\right)$ can easily be verified for any $m \leqslant n<\omega$. Therefore,

$$
d(u, v)=d\left(u, z_{1}\right)+d\left(z_{1}, z_{2}\right)+\cdots+d\left(z_{n-1}, z_{n}\right)+d\left(z_{n}, v\right) \geqslant n \cdot d(x, y)
$$

for each $n<\omega$. As $d(x, y)>0, d$ cannot take values in an Archimedean ordered field.

(2) $\neg \theta \notin$ Th $\mathcal{M}$ : It suffices to find an $(A, T) \in \mathcal{M}$ satisfying $\theta$. Let $A$ consist of the chain $u=z_{0}<z_{1}<\cdots<z_{n}<z_{n+1}<\cdots<z_{\omega}=v$, ordered by the type $\omega+1$, and two other elements $x, y$. Let $T$ contain all the triples of the form $(a, a, b)$ $(a, b \in A),\left(x, y, z_{2 n+1}\right),\left(z_{2 n}, x, y\right)(n<\omega),\left(z_{m}, z_{n}, z_{\alpha}\right)(m<n<\alpha \leqslant \omega)$, and the reversed ones - see Diagram 1.

One can check that $(A, T)$ satisfies $\theta$. On the other hand, $(A, T)$ is metrizable in any non-Archimedean ordered field $F$. Indeed, identifying $n<\omega$ with $n \cdot 1 \in F$ and $\omega$ with an arbitrary element of $F$ bigger than any $n$, the metric $d: A \times A \rightarrow F$ can be defined by, say,

$$
\begin{gathered}
d(x, y)=1, \quad d\left(z_{m}, z_{n}\right)=2|m-n|, \quad d\left(z_{n}, v\right)=\omega-2 n, \\
d\left(x, z_{2 n}\right)=d\left(y, z_{2 n+1}\right)=d(x, v)=d(y, v)=\omega, \quad d\left(x, z_{2 n+1}\right)=d\left(y, z_{2 n}\right)=\omega+1,
\end{gathered}
$$

for all $m, n<\omega$. Then $T=T_{d}$ is plain.

Thus we see that $\mathcal{M}$ is not the least elementary class containing $\mathcal{M}_{0}$. Nevertheless, $\mathcal{M}$ is still a certain closure of $\mathcal{M}_{0}$.

Theorem 2. $\mathcal{M}$ is both the least universal class and the least universal-existential class containing $\mathcal{M}_{0}$, i.e., $\operatorname{Th}_{\forall} \mathcal{M}_{0}=\operatorname{Th}_{\forall \exists} \mathcal{M}_{0}=\operatorname{Th} \mathcal{M}$.

Proof. Let $\mathcal{K}$ denote the least universal-existential class containing $\mathcal{M}_{0}$. Obviously, $\mathcal{K} \subseteq \mathcal{M}$. It suffices to show that every countable $(A, T) \in \mathcal{M}$ belongs to $\mathcal{K}$. Each $(A, T)$ can be written as the union of a chain of its finite substructures. Since $\mathcal{M}$ and $\mathcal{M}_{0}$ contain the same finite structures and the universal-existential class $\mathcal{K}$ is closed under chain unions, $(A, T) \in \mathcal{K}$. Hence $\operatorname{Th}_{\forall \exists}=\mathcal{M}_{0}=\operatorname{Th} \mathcal{M}$. Since $\operatorname{Th} \mathcal{M} \subset \operatorname{Th} \mathcal{M}_{0}$, we have $\operatorname{Th}_{\forall} \mathcal{M} \subseteq \operatorname{Th}_{\forall} \mathcal{M}_{0}$, therefore, since $\operatorname{Th}_{\forall} \mathcal{M}=\operatorname{Th} \mathcal{M}$ (by part (ii) of the Theorem in [1]), $\operatorname{Th} \mathcal{M} \subseteq \mathrm{Th}_{\forall} \mathcal{M}_{0}$. The statement of the theorem now follows from $\operatorname{Th}_{\forall} \mathcal{M}_{0} \subseteq \operatorname{Th}_{\forall \exists} \mathcal{M}_{0}=\operatorname{Th} \mathcal{M}$. 
As $\neg \theta$ is equivalent to an existential-universal sentence, the counterexample of Theorem 1 is the best possible.

\section{REFERENCES}

[1] R. Mendris and P. Zlatoš, Axiomatization and undecidability results for metrizable betweenness relations, Proc. Amer. Math. Soc. 123 (1995), 873-882. MR 95d:03008

Department of Mathematics, Faculty of Chemical Technology, Slovak Technical University, 81237 Bratislava, Slovakia

E-mail address: simko@cvt.stuba.sk 